\title{
Heberprot-P's Effect on Gene Expression in Healing Diabetic Foot Ulcers
}

\author{
Hanlet Camacho-Rodríguez MS, Isabel A. Guillen-Pérez PhD, Juan Roca-Campaña MD, Julio E. Baldomero-Hernández MD, \\ Ángela D. Tuero-Iglesias, José A. Galván-Cabrera MD PhD, Maciel Rodriguez-Cordero, Daniel O. Palenzuela-Gardón MS, \\ Jorge Berlanga-Acosta DVM MS PhD, Lidia I. Novoa-Pérez PhD
}

\begin{abstract}
INTRODUCTION Diabetic foot ulcers are a chronic complication in patients with diabetes mellitus. They appear as a result of the combination of diabetic polyneuropathy and angiopathy, and in many cases require amputation of the affected extremity. Clinical trials have demonstrated that repeated local infiltration with Heberprot-P can improve healing of chronic diabetic foot ulcers. Although there is evidence of its effects as a granulation stimulator and on cell migration and proliferation, genetic control mechanisms explaining its anti-inflammatory and oxidative stress reduction properties are not yet thoroughly understood.
\end{abstract}

OBJECTIVE Analyze changes in expression of genes involved in healing after treatment of diabetic foot ulcers with Heberprot-P.

METHODS Biopsies were collected from diabetic foot ulcers of 10 responding patients before and after 2 weeks' treatment with Heberprot-P (75- $\mu \mathrm{g}$ applied intralesionally 3 times per week). Total RNA was obtained and quantitative PCR used to determine expression of 26 genes related to inflammation, oxidative stress, cell proliferation, angiogenesis and extracellular matrix formation. Genetic expression was quantified before and after treatment using REST 2009 v2.0.13.

RESULTS After treatment, there was a statistically significant increase in expression of genes related to cell proliferation, angiogenesis and formation of extracellular matrix (PDGFB, CDK4, P21, TP53, ANGPT1, COL1A1, MMP2 and TIMP2). A significant decrease was observed in gene expression related to inflammatory processes and oxidative stress (NFKB1, TNFA and IL-1A).

CONCLUSIONS Our findings suggest that Heberprot-P's healing action on diabetic foot ulcers is mediated through changes in genetic expression that reduce hypoxia, inflammation and oxidative stress, and at the same time increase cell proliferation, collagen synthesis and extracellular matrix remodeling. The kinetics of expression of two genes related to extracellular matrix formation needs further exploration.

KEYWORDS Epidermal growth factor, EGF, diabetic foot ulcer, wound healing, quantitative real-time PCR, gene expression, Cuba

\section{INTRODUCTION}

Diabetes mellitus (DM) is a chronic disease with increasing global prevalence over recent decades; according to $\mathrm{WHO}$, DM affects $8.5 \%$ of the global population.[1] One of its main complications is lower-extremity ulceration, known as diabetic foot ulcer (DFU), which often leads to amputation.[2,3] Recent reports on DM in Cuba suggest an overall prevalence of 58.3 per 1000 population.[4]

Diabetes-induced hyperglycemia activates four biochemical pathways: the polyol, hexosamine, protein-kinase $C(P K C)$ and advanced glycation end products (AGE). Together, these cause inflammation and oxidative stress (OS).[5,6]

Endothelial cells in the vasculature, neurons and Schwann cells in peripheral nerves contain only high-affinity glucose transporter proteins (GLUT1 and GLUT3).[7] Thus, in hyperglycemic conditions, a massive and unregulated amount of glucose enters these cells, which makes them targets for inflammation and OS, and explains the occurrence of long-term complications such as diabetic angiopathy and polyneuropathy-the main causes of DFU.[8,9]

IMPORTANCE This research increases our understanding of the complex mechanisms of action by which Heberprot-P speeds wound healing in diabetic foot ulcers, reducing related amputations and mortality.
Wound healing is the process by which damaged tissue is replaced by healthy connective tissue, forming a scar. This process can be divided into four dynamic, overlapping phases: vascular response, inflammatory response, proliferation and maturation (or remodeling).[10]

According to estimates from Berlanga in 2013, 3000 to 5000 amputations are performed annually in Cuba due to DFU. To treat DFU, the Genetic Engineering and Biotechnology Center in Havana developed Heberprot-P, based on human recombinant epidermal growth factor (EGF).[11]

Local conditions resulting from hyperglycemia in DM include:

- decreased vascularization from a reduction in expression of genes regulating angiogenesis, namely vascular endothelial growth factor (VEGF) and angiopoietin-1. This causes hypoxia and cytoplasmic membrane rupture leading to release of cellular content, increased inflammation and OS.

- increased chronic inflammation and OS. These are linked to diabetic angiopathy and polyneuropathy and are a consequence of increased expression of proinflammatory cytokine genes, including tumor necrosis factor alpha (TNFA), interleukin 6 (IL-6), and interleukin 1 alpha (IL-1A). There is also increased expression of genes for the receptor for advanced glycation end products (AGER), related to OS, as well as the gene that regulates their expression, NF-kappa $B$ transcription factor (NFKB1).

- reduced bioavailability of growth factors due to the excess of proteases released by active neutrophils. There are five families of growth factor: EGF, platelet-derived growth factor 
(PDGF), transforming growth factor beta (TGFB), insulin-like growth factor (IGF) and fibroblast growth factor (FGF). They all play a part in healing and the processes of chemotaxis, cell proliferation induction, angiogenesis stimulation, synthesis regulation and extracellular matrix (ECM) degradation. Prolonged inflammation prevents progression to the proliferation phase and causes delayed or incomplete healing. $[12,13]$

Although gene expression can be controlled at various levels, it is widely accepted that it generally happens in DNA transcription, and evidence of degree of a gene's expression can be observed by measuring the quantity of messenger RNA corresponding to the gene's DNA.[14,15] To study gene expression variation, real-time PCR is routinely used in molecular biology to amplify products transcribed from messenger RNA. Quantification of such variation may be relative (based on target gene expression relative to that of a reference gene) or absolute (based on an internal or external calibration curve). With relative quantification, change in RNA expression is shown as the fold change between two sample groups using normalization, a process that compares the degree of expression of the genes being studied with two or more reference genes that have unchanging expression levels, regardless of cell type and treatment being investigated.[16]

It has been reported that treatment with Heberprot-P leads to a $77 \%$ cure rate in cases of DFU,[17] and that EGF stimulates proliferation of epithelial cells, fibroblasts and vascular endothelial cells.[13] However, there is little information regarding which changes in gene expression could lead to ulcer healing in patients with DFU treated with EGF.

This study's goal was to analyze changes in gene expression involved in processes affecting DFU healing (inflammation and OS, cell proliferation, angiogenesis, and ECM formation and remodeling) after treatment with Heberprot-P and clinical evidence of patient response.

\section{METHODS}

Design Ulcerous tissue was biopsied in 156 patients included in clinical trial code IG/FCEI/PD/0911 in the Cuban Public Registry of Clinical Trials, prior to treatment (T0) with Heberprot-P (75- $\mu$ g dose applied intralesionally, 3 times per week). Another biopsy was taken after 2 weeks of application (T1) in granulation tissue. At the end of the study, 29 patients met the following criteria: they had been treated for Wagner grade 3-4 diabetic foot ulcers, they responded to treatment with Heberprot-P,[2] and their RNA samples were of optimal quality for differential expression studies.[18] Of the 29 patients who met study criteria, 10 were chosen at random, the minimum sample size able to detect a 1.5 -fold difference with $80 \%$ statistical power and a maximum of $5 \%$ type I error. [19] Patients were considered responders if they had complete wound closure at end of treatment with Heberprot-P.

Relative expression of genes of interest was measured by comparing expression levels in biopsies taken at T1 vs. T0. The experiments were normalized using previously validated reference genes as internal controls, each group with a total of 10 biological replicates; 3 technical replicates were used for each gene. A significance threshold of $p=0.05$ was chosen.
RNA purification Extracted samples were stored in Ambion RNAlater (AppliedBiosystems, USA) at $-20^{\circ} \mathrm{C}$ for one week. Tissue was processed in a Tissue Lyser unit (Qiagen, Germany). Total RNA was extracted with the RNeasy Plus reagent kit (Qiagen $\mathrm{GmbH}$, Germany) using the Quiacube platform (Qiagen, Germany).

RNA quality control Quantity, purity and integrity of RNA was assessed using the Nano Drop spectrophotometer (Thermo Fisher Scientific, USA) and the Bioanalizador Agilent 2100 with the Eukaryote RNA 6000 Nano Chip (Agilent Technologies, USA). RNA integrity values greater than seven are considered acceptable for differential gene expression studies.[18]

Complementary DNA synthesis The complementary DNA chain was synthesized from $1 \mu \mathrm{g}$ of total RNA using Superscript III First-Strand Synthesis Supermix for qRT-PCR (Invitrogen Technologies, USA), per manufacturer's instructions.

qPCR and bioinformatics tools Gene sequence expression was obtained from the US National Center for Biotechnology Information database (Table 1).[20] Specific primers were designed for amplification of genes of interest, using the web application Primer3.[21] Reference genes were selected from a group of candidate genes using the geNorm tool.[22] qPCR reactions were incubated in an optical detection rotor (Capital Bio Co., China) and prepared using the Thermo Scientific ABsolute QPCR SYBR Green Mix reagent case (Thermo Fisher Scientific, USA), per manufacturer's instructions. The qPCR data was analyzed using the Capital Bio RT-Cycler analysis program, version 2.001 (Capital Bio Co. Ltd., China) and relative quantification of genetic expression was performed using REST 2009 v2.0.13.[23] Differences were expressed as fold changes.

Ethics Samples used in this study were from a clinical trial (code IG/FCEI/PD/0911, approved by Cuba's Center for State Control of Medicines and Medical Devices, registration number Reg/10/002/Z/SAEC/01, results not yet published). Participating patients gave written informed consent according to Declaration of Helsinki principles.[24]

\section{RESULTS}

Quality control performed with Bioanalyzer and Nanodrop complied with accepted parameters for RNA sample use in differential gene expression studies.[18] Average RNA concentration was 468.96 ng/uL (SD 308.57) at T0 and 669.08 ng/uL (SD 365.24) at T1. RNA integrity was 8.22 (DS 0.82 ) at T0 and 8.2 (DS 0.9 ) at T1.

Comparing DFU patients' biopsies at T1 to those at T0 revealed an increase in expression of genes related to cell proliferation (CDK4, CDKN1B, P21, TP53 and FOS); differences were statistically significant for CDK4, P21 and TP53 (Table 2). There was also increased expression of genes involved in collagen synthesis and ECM remodeling, (COL1A1, MMP2, MMP7, MMP9, TIMP1 and TIMP2). Increases were statistically significant for COL1A1, MMP2 and TIMP2 (Table 2). Decreases were detected for genes related to inflammation and OS (IL-1a, IL-6, IL-17, TNFA, NFKB1 and AGER), statistically significant for NFKB, TNFA and IL-1A, but not for IL17, IL6 and AGER (Table 2).

Expression increased for another group of genes related to proliferation and cell migration-protein-3 insulin-like growth factor- 
Table 1: Genes analyzed by qPCR

\begin{tabular}{|c|c|c|}
\hline Gene & Access \# & Biological function \\
\hline AGER & NM_001136.4 & AGE receptor \\
\hline ANGPT1 & NM_001146.3 & Transcription factor \\
\hline CDK4 & NM_000075 & Cell cycle \\
\hline CDKN1B & NM_004064.4 & Cell cycle \\
\hline COL1A1 & NM_000088.3 & Collagen protein \\
\hline CTGF & NM_001901.2 & Growth factor \\
\hline FOS & NM_005252.3 & Transcription factor \\
\hline HIFA1 & NM_001243084.1 & Transcription factor \\
\hline IGFBP3 & NM_001013398.1 & Cell proliferation \\
\hline IL-17 & NM_002190.2 & Proinflammatory cytokine \\
\hline IL-1A & NM_000575.4 & Proinflammatory cytokine \\
\hline IL-6 & NM_000600.4 & Proinflammatory cytokine \\
\hline MMP2 & NM_004530.5 & Tissue remodeling \\
\hline MMP7 & NM_002423.4 & Tissue remodeling \\
\hline MMP9 & NM_004994.2 & Tissue remodeling \\
\hline NFKB1 & NM_003998.3 & Transcription factor \\
\hline P21 (WAF1) & NM_000389.4 & Cell cycle \\
\hline PDGFB & NM_002608.2 & Growth factor \\
\hline PHB & NM_002634.2 & Transcription factor \\
\hline PLCG1 & NM_002660.2 & Membrane associated enzyme \\
\hline TGFB1 & NM_000660.3 & Growth factor \\
\hline TIMP1 & NM_003254.2 & Tissue remodeling \\
\hline TIMP2 & NM_003255.4 & Tissue remodeling \\
\hline TNFA & NM_000594.3 & Proinflammatory cytokine \\
\hline TP53 & NM_000546.4 & Transcription factor \\
\hline VEGFA & NM_001025366.1 & Growth factor \\
\hline GAPDH & NM_001256799.2 & Reference gene \\
\hline MAP2K5 & NM_001206804.1 & Reference gene \\
\hline MAPK6 & NM_002748.3 & Reference gene \\
\hline RPL13A & NM_001270491.1 & Reference gene \\
\hline YWHAZ & NM_001135699.1 & Reference gene \\
\hline
\end{tabular}

AGE: advanced glycation end product

Source: US NCBI[20]

binding factor (IGFBP3) and PDGFB—but the increase was only statistically significant for PDGFB. Prohibitin (PHB) expression decreased, but not significantly.

There was increased expression of VEGFA and ANGPT1-genes related to angiogenesis and ischemia-the latter statistically significant, while there was reduced expression of hypoxia-inducible factor 1, alpha subunit (HIF1A). There was also decreased expression of TGFB 1 and connective tissue growth factor (CTGF), genes related to ECM formation; and of phospholipase $C$, gamma 1 protein, (PLCG1), genes related to the PKC pathway (Table 2).

\section{DISCUSSION}

A proposed conceptual model of Heberprot-P's mechanism is displayed in Figure 1.

Biochemical mechanisms suggested for diabetic neuropathy's etiology include nonenzymatic glycosylation with AGE formation and activation of the PKC pathway, which cause both inflammation and OS. They also contribute to damage in nerve, glial and
Table 2: Change in gene expression after treatment with Heberprot-P

\begin{tabular}{r|l|r|c|c|}
\hline No. & Gene & Fold change & P-value & $\begin{array}{c}\text { Direction of } \\
\text { change* }\end{array}$ \\
\hline 1 & AGER & -1.20 & 0.190 & \\
\hline 2 & ANGPT1 & 1.45 & 0.001 & $\uparrow$ \\
\hline 3 & CDK4 & 1.48 & 0.009 & $\uparrow$ \\
\hline 4 & CDKN1B & 1.04 & 0.568 & \\
\hline 6 & COL1A1 & 1.67 & 0.005 & $\uparrow$ \\
\hline 7 & CTGF & -1.28 & 0.302 & \\
\hline 8 & FOS & 1.10 & 0.740 & \\
\hline 9 & IGF1A & -1.25 & 0.088 & \\
\hline 10 & IL17A & 1.28 & 0.220 & \\
\hline 11 & IL-1A & -2.17 & 0.079 & \\
\hline 12 & IL-6 & -13.70 & 0.000 & $\downarrow$ \\
\hline 13 & MMP2 & -1.78 & 0.207 & \\
\hline 14 & MMP7 & 2.21 & 0.000 & $\uparrow$ \\
\hline 15 & MMP9 & 1.07 & 0.886 & \\
\hline 16 & NFKB1 & 1.69 & 0.090 & \\
\hline 17 & P21 & -1.37 & 0.002 & $\downarrow$ \\
\hline 18 & PDGFB & 1.54 & 0.009 & $\uparrow$ \\
\hline 19 & PHB & 1.68 & 0.002 & $\uparrow$ \\
\hline 20 & PLCG1 & -1.20 & 0.073 & \\
\hline 21 & TGFB1 & -1.08 & 0.325 & \\
\hline 22 & TIMP1 & -1.08 & 0.540 & \\
\hline 23 & TIMP2 & 1.08 & 0.652 & \\
\hline 24 & TNFA & 1.43 & 0.007 & \\
\hline 25 & TP53 & -1.96 & 0.001 & \\
\hline 26 & VEGFA & 1.99 & 0.000 & \\
\hline & 1.38 & 0.227 & \\
\hline
\end{tabular}

*where significant

vascular endothelial cells, causing diabetic angiopathy and polyneuropathy.[25]

AGE molecules can spread outside cells and modify blood proteins such as albumin. By binding to specific AGERs, these modified proteins activate the NFKB1 pathway, which induces expression of proinflammatory cytokines and increases production of reactive oxygen species. NFKB1 also controls AGER expression.[25,26]

The significant decrease at T1 in expression of the transcription factor NFKB1 is associated with reduced expression of proinflammatory cytokines and AGER genes. This implies, in turn, less damage from inflammation and OS. The lack of statistical significance for the reduced expression of proinflammatory cytokines genes IL 6 and IL17 can be explained by data dispersion.

The PLCG1 enzyme catalyzes formation of diacylglycerol (DAG), a PKC pathway activator.[27] Therefore, the observed decrease in PLCG1 expression (Table 2) may have prevented activation of the PKC pathway, an important mechanism in the physiopathology of diabetic complications.

Increased expression of VEGFA and ANGPT1 genes (the latter significantly) favors angiogenesis, and is related to decreased expression of HIF1A, a transcription factor expressed in tissue hypoxia (Table 2). This increased blood flow may promote DFU healing. 
Figure 1: Heberprot-P mechanisms of action

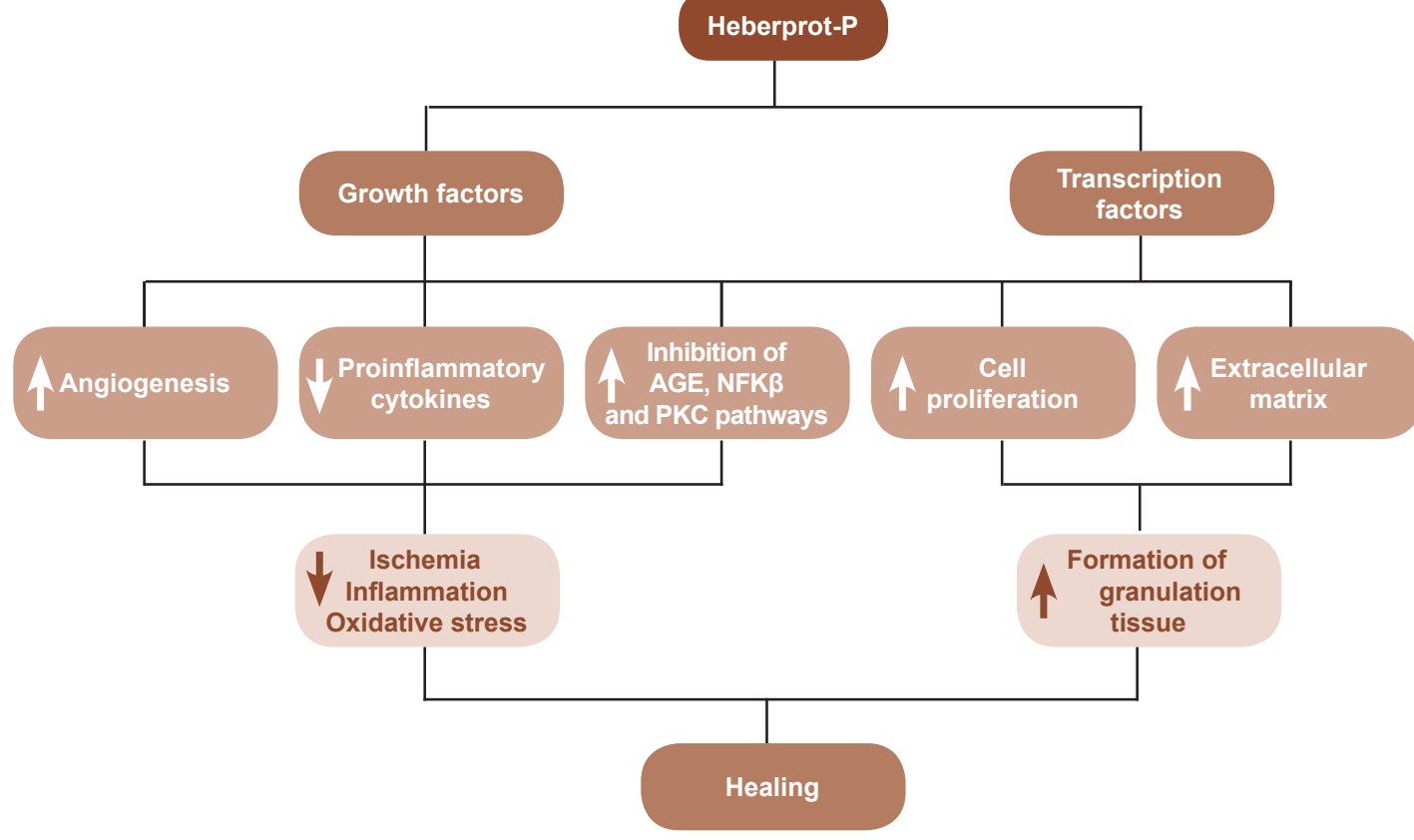

Increased MMP expression thus implies a poor prognosis for ulcer healing. At T1, when there is decreased expression of inflammatory genes and formation of granulation tissue, increased expression of MMP genes could suggest that healing is at a more advanced stage, because MMP acts to remodel the scar tissue being formed,[35] and thus its increase could be interpreted differently than as proposed by Liu.[33]

The decreased expression of TGFB1 and CTGF, which stimulate expression of genes related to ECM formation, is paradoxical,[36,37] and could be due to the fact that both genes are expressed transiently, with maximum expression in early healing.

Increased expression of PDGFB, a potent cell proliferation stimulator[28,29] and the resulting decrease of PHB, a negative regulator of proliferation,[30,31] may explain the increased expression of genes related to the cell cycle. We also observed an increase in expression of IGBP3, which according to Ferry, regulates bioavailability of another growth factor, IGF.[32]

The heightened expression in responders of genes involved in ECM formation and remodeling (Table 2), specifically MMP genes, may seem to contradict Liu's findings; Liu suggests that increased MMP9 predicts poor DFU healing through its association with inflammation.[33] However, it has also been reported that MMP2 and MMP9 can be produced by fibroblasts and keratinocytes, which are noninflammatory cells, and their functions could be different in a repair microenvironment.[34]

Healing goes through several phases. At T0, the DFU is in the inflammation phase, when increased expression of MMP genes (which degrade components of ECM and basement membrane proteins) causes serious tissue damage, suppressing reepithelialization.
$[38,39]$ At T0, there is inflammation and therefore there should be heightened expression of TGFB1 and CTGF. At T1, there is resolution of inflammation and healing is in the proliferation and remodeling phase. Therefore, one might well expect a relative decrease in TGFB1 and CTGF gene expression at T1.

One limitation of this study is that analysis of gene expression was performed with biopsies at only two points in the ulcer healing process, insufficient to detect early expression of genes. Despite this, and limited sample size, our results offer a clearer view of transcriptional activity induced by Heberprot-P in responders with DFU.

\section{CONCLUSION}

Our findings suggest that Heberprot-P's DFU healing action is poxia, inflammation and oxidative stress, and increase cell proliferation, collagen synthesis and ECM remodeling. The kinetics of expression of two genes related to ECM formation needs further exploration. -1 mediated through changes in genetic expression that reduce hy-

\section{REFERENCES}

1. World Health Organization. Global Report on Diabetes [Internet]. Geneva: World Health Organization; 2016 [cited 2017 Jan 25]. 86 p. Available from: http://apps.who.int/iris/bitstream/ handle/10665/204871/9789241565257_eng.pdf; jsessionid=6D909E47C95E30D51430F445A8F7 $592 E$ ? sequence $=1$

2. Clayton $\mathrm{W} \mathrm{Jr}$, Elasy TA. A review of the pathophysiology, classification, and treatment of foot ulcers in diabetic patients. Clin Diabetes. 2009 Apr;27(2):52-8.

3. Reiber GE, Vileikyte L, Boyko EJ, del Aguila M, Smith DG, Lavery LA, et al. Causal pathways for incident lower extremity ulcers in patients with diabetes from two settings. Diabetes Care. 1999 Jan;22(1):157-62.

4. National Health Statistics and Medical Records Division (CU). Anuario Estadístico de Salud
2016. Havana: Ministry of Public Health (CU); 2017 Apr. 206 p. Spanish.

5. Giacco F, Brownlee M. Oxidative stress and diabetic complications. Circ Res. 2010 Oct 29;107(9):1058-70.

6. Brownlee M. Biochemistry and molecular cell biology of diabetic complications. Nature. 2001 Dec 13;414(6865):813-20.

7. Bermúdez $V$, Bermúdez $F$, Arraiz $N$, Leal E, Linares S, Mengua E, et al. Biología molecular de los transportadores de glucosa: clasificación, estructura y distribución. Arch Venezolanos Farmacol Terap. 2007;26(2):7685. Spanish.

8. Papanas N, Maltezos E. Etiology, pathophysiology and classifications of the diabetic Charcot foot. Diabet Foot Ankle. 2013 May 21;4. DOI: 10.3402/dfa.v4i0.20872
9. Forbes JM, Cooper ME. Mechanism of diabetic complication. Physiol Rev. 2013 Jan;93(1):137-88.

10. Flanagan $M$. The physiology of wound healing. $J$ Wound Care. 2000 Jun;9(6):299-300.

11. Berlanga J, Fernández JI, López $\mathrm{E}$, López PA del Río A, Valenzuela $C$, et al. Heberprot-P: a novel product for treating advanced diabetic foot ulcer. MEDICC Rev. 2013 Jan;15(1):11-5.

12. Zhao $R$, Liang $H$, Clarke $E$, Jackson $C$, Xue $M$. Inflammation in chronic wounds. Int $\mathrm{J}$ Mol Sci. 2016 Dec 11;17(12). pii: E2085.

13. Traversa B, Sussman G. The role of growth factors, cytokines and proteases in wound management. Growth factors, cytokines \& proteases. 2001 Nov;9(4):161-7.

14. Lodish H, Berk A, Matsudaira P, Kaiser C. Molecular Cell Biology. 5th ed. New York: W. H. Freeman; 2003 Aug 1. 973 p. 
15. Berg JM, Tymoczko JL, Stryer L. Biochemistry. 5th ed. New York: W. H. Freeman; 2002 Feb 15. $1100 \mathrm{p}$.

16. Pfaffl MW. A new mathematical model for relative quantification in real-time RT-PCR. Nucleic Acids Res. 2001 May 1;29(9):e45.

17. Fernández-Montequín JI, Valenzuela-Silva CM, González-Díaz O, Savigne W, Sancho-Soutelo $\mathrm{N}$, Rivero-Fernández $\mathrm{F}$, et al. Intra-lesional injections of recombinant human epidermal growth factor promotes granulation and healing in advanced diabetic foot ulcers. Multicenter, randomized, placebo-controlled, double blind study. Int Wound J. 2009 Dec;6(6):432-43.

18. Fleige S, Pfaffl MW. RNA integrity and the effect on the real-time qRT-PCR performance. Mol Aspects Med. 2006 Apr-Jun;27(2-3):126-39.

19. Multid Analyses $A B$ [Internet]. Göteborg (SE): MultiD Analyses AB; c2001-2015. GenEX 6.1 software; 2016 Feb 29 [cited 2017 May 27]. Available from: http://www.multid.se

20. National Center for Biotechnology Information [Internet]. Bethesda (US): U.S. National Library of Medicine. Available from: https://www.ncbi .nlm.nih.gov/

21. Rozen S, Skaletsky HJ. Primer3 on the WWW for general users and for biologist programmers. Methods Mol Biol. 2000;132:365-86.

22. Vandesompele J, De Preter K, Pattyn F, Poppe B, Van Roy N, De Paepe A, et al. Accurate normalization of real-time quantitative RT-PCR data by geometric averaging of multiple internal control genes. Genome Biol. 2002 Jun 18;3(7):RESEARCH0034. Epub 2002 Jun 18.

23. Pfaffl MW, Horgan GW, Dempfle L. Relative expression software tool (REST) for group-wise comparison and statistical analysis of relative expression results in real-time PCR. Nucleic Acids Res. 2002 May 1;30(9):e36.

24. World Medical Association. Declaration of Helsinki: ethical principles for medical research involving human subjects. JAMA [Internet]. $2013 \mathrm{Nov}$ 27 [cited 2018 May 5];310(20):2191-4. Available from: https://jamanetwork.com/journals/jama/full article/10.1001/jama.2013.281053

25. Brownlee M. The pathobiology of diabetic complications: a unifying mechanism. Diabetes. 2005 Jun;54(6):1615-25.

26. Goldin A, Beckman JA, Schmidt AM, Creager MA. Advanced glycation end products: sparking the development of diabetic vascular injury. Circulation. 2006 Aug 8;114(6):597-605.

27. Noh $\mathrm{H}$, King $\mathrm{GL}$. The role of protein kinase $\mathrm{C}$ activation in diabetic nephropathy. Kidney Int Suppl. 2007 Aug;(106):S49-S53.
28. Pierce GF, Mustoe TA, Altrock BW, Deuel TF, Thomason A. Role of platelet-derived growth factor in wound healing. J Cell Biochem. 1991 Apr:45(4):319-26. DOI:10.1002./jcb.240450403.

29. Werner S, Grose R. Regulation of wound healing by growth factors and cytokines. Physiol Rev. 2003 Jul;83(3):835-70.

30. Wang S, Nath N, Fusaro G, Chellappan S. Rb and prohibitin target distinct regions of E2F1 for repression and respond to different upstream signals. Mol Cell Biol. 1999 Nov;19(11):7447-60.

31. Mishra S, Murphy LC, Murphy LJ. The Prohibitins: emerging roles in diverse functions. J Cellular Molecular Med. 2006;10(2):353-63.

32. Ferry RJ Jr, Katz LE, Grimberg A, Cohen P, Weinzimer SA. Cellular actions of insulin-like growth factor binding proteins. Horm Metab Res. 1999 Feb-Mar;31(2-3):192-202.

33. Liu $Y$, Min D, Bolton T, Nubé $V$, Twigg SM, Yue $\mathrm{DK}$, et al. Increased matrix metalloproteinase-9 predicts poor wound healing in diabetic foot ulcers. Diabetes Care. 2009 Jan;32(1):117-9.

34. Bhupinder S. Matrix metalloproteinase: an overview. Res Reports Biol. 2010 Sep 14;1:1-20.

35. Armstrong DG, Jude EB. The role of matrix metalloproteases in wound healing. J Am Podiatric Med Assoc. 2002 Jan;92(1):12-8.

36. Frazier K, Williams S, Kothapalli D, Klapper H, Grotendorst GR. Stimulation of fibroblast cell growth, matrix production, and granulation tissue formation by connective tissue growth factor. J Invest Dermatol. 1996 Sep;107(3):404-11.

37. Pakyari M, Farrokhi A, Maharlooei MK, Ghahary A. Critical role of transforming growth factor beta in different phases of wound healing. Adv Wound Care (New Rochelle). 2013 Jun;2(5):215-24.

38. Werner S, Grose R. Regulation of wound healing by growth factors and cytokines. Physiol Rev. 2003 Jul;83(3):835-70.

39. Deonarine K, Panelli MC, Stashower ME, Jin P, Smith K, Slade HB, et al. Gene expression profiling of cutaneous wound healing. J Transl Med. 2007 Feb 21;5:11.

\section{THE AUTHORS}

Hanlet Camacho-Rodríguez (Corresponding author: hamlet.camacho@cigb.edu.cu), microbiologist with a master's degree in neurosciences. Associate researcher, Biomedical Research Division (DIB), Center for Genetic Engineering and Biotechnology (CIGB), Havana, Cuba.
Isabel A. Guillén-Pérez, biologist. Senior researcher, DIB, CIGB, Havana, Cuba.

Juan Roca-Campaña, physician specializing in clinical biochemistry, DIB, CIGB, Havana, Cuba.

Julio E. Baldomero-Hernández, physician specializing in family medicine, Clinical Research Division (DIC), CIGB. Havana, Cuba.

Ángela Tuero-Iglesias, mathematician. Associate researcher, DIC, CIGB. Havana, Cuba.

José A. Galván-Cabrera, physician specializing in clinical biochemistry with a doctorate in medical sciences, Immunology Department, Hematology and Immunology Institute, Havana, Cuba.

Maciel Rodríguez-Cordero, biochemist. Research fellow, DIB, CIGB. Havana, Cuba.

Daniel O. Palenzuela-Gardón, physicist with a master's degree in new product research. Associate researcher, DIB, CIGB. Havana, Cuba

Jorge Berlanga-Acosta, veterinarian with a master's degree in pathology and a doctorate in pharmacology, DIB, CIGB. Havana, Cuba

Lidia I. Novoa-Pérez, biologist. Senior researcher, DIB, CIGB. Havana, Cuba

Submitted: October 27, 2017

Approved for publication: July 2, 2018

Disclosures: All authors except Galván-

Cabrera are employed at CIGB, developer of Heberprot- $P$ 